\title{
Quality indicators in headache care: an implementation study in six Italian specialist-care centres
}

\author{
L. Pellesi ${ }^{1 *}$, S. Benemei ${ }^{2}$, V. Favoni ${ }^{3}$, C. Lupi ${ }^{2}$, E. Mampreso ${ }^{4}$, A. Negro ${ }^{5}$, M. Paolucci ${ }^{6}$, T. J. Steiner ${ }^{7,8}$, M. Ulivi ${ }^{6}$,
}

S. Cevoli ${ }^{9}$ and S. Guerzoni ${ }^{1}$

\begin{abstract}
Background: Headache disorders are highly prevalent, and have a substantial and negative impact on health worldwide. They are largely treatable, but differences in structure, objectives, organization and delivery affect the quality of headache care. In order to recognize and remedy deficiencies in care, the Global Campaign against Headache, in collaboration with the European Headache Federation, recently developed a set of quality indicators for headache services. These require further assessment to demonstrate fitness for purpose. This is their first implementation to evaluate quality in headache care as a multicentre national study.

Methods: Between September and December 2016, we applied the quality indicators in six Italian specialist headache centres (Bologna, Firenze, Modena, Padova, Roma Campus Bio-Medico and Roma Sapienza). We used five previously developed assessment instruments, translated into Italian according to Lifting The Burden's translation protocol for hybrid documents. We took data from 360 consecutive patients (60 per centre) by questionnaire and from their medical records, and by different questionnaires from their health-care providers (HCPs), including physicians, nurses, psychologists and nursing assistants.

Results: The findings, comparable between centres, confirmed the feasibility and practicability of using the quality indicators in Italian specialist headache centres. The questionnaires were easily understood by HCPs and patients, and were not unduly time-consuming. Diagnoses were almost all (> 97\%) according to ICHD criteria, and routinely (100\%) reviewed during follow-up. Diagnostic diaries were regularly used by $96 \%$ of physicians. Referral pathways from primary to specialist care existed in five of the six clinics, as did urgent referral pathways. Instruments to assess disability and quality of life were not used regularly, a deficiency that needs to be addressed.

Conclusion: This Italy-wide survey confirmed in six specialist centres that the headache service quality indicators are fit for purpose. By establishing majority practice, identifying commonalities and detecting deficits as a guide to quality improvement, the quality indicators may be used to set benchmarks for quality assessment. The next step is extend use and evaluation of the indicators into non-specialist care.
\end{abstract}

Keywords: Headache disorders, Headache care, Service quality evaluation, Benchmarking, Global Campaign against Headache

\footnotetext{
* Correspondence: lanfranco.pellesi@gmail.com

'Medical Toxicology - Headache Centre, Policlinic Hospital, University of

Modena and Reggio Emilia, Modena, Italy

Full list of author information is available at the end of the article
} 


\section{Background}

Headache disorders are highly prevalent, with major negative impacts on health, well-being and productivity worldwide. They are treatable, but differences in structure, objectives, organization and delivery affect the quality of medical care provided for them. While service quality is one of the most important factors influencing outcomes delivered by a health system, "quality" does not have clear meaning in this context, and its measurement is not straightforward. In the field of headache disorders, although systematic classification and diagnostic criteria have been in place since 1988 [1], no definition of service quality existed until recently [2]. Lacking the grounding of any such definition, quality indicators proposed in the past have been partial and selective, and assessed for neither validity nor usability across headache-care settings [2].

The Global Campaign against Headache [3, 4], conducted by Lifting the Burden (LTB), a UK-registered non-governmental organization in official relations with the World Health Organization, aims inter alia to remedy deficiencies in headache-care worldwide. This requires a means of identifying such deficiencies. In a collaborative project with the European Headache Federation (EHF), LTB used various qualitative research methods to develop, through focus-group consultations and expert consensus, a definition of quality applicable to headache services.

\section{"Good-quality headache care achieves accurate diagnosis and individualized management, has appropriate referral pathways, educates patients about their headaches and their management, is convenient and comfortable, satisfies patients, is efficient and equitable, assesses outcomes and is safe" [5].}

Along with this definition, LTB proposed 30 indicators in nine quality domains that might be used to evaluate headache service quality, with the objectives not only identifying deficiencies but also of guiding their remedy. Each was associated with a specific assessment instrument.

The indicators were developed to be applicable across countries and cultures, and at all levels within health systems, in primary care and in specialist centres. No external standards were available to assess criterionvalidity, which depended therefore on the methodological rigour employed in their development [5]. In a pilot implementation study conducted successfully in two specialist headache centres in Portugal and Germany [6], the assessment questionnaires were found easy to apply by health-care practitioners (HCPs) and patients, and not unduly time consuming [6]. Despite the differences in language and institutions (a university teaching hospital versus a private hospital), the findings were comparable in the two countries, suggesting operational validity in the evaluation of headache care. A subsequent pan-European study confirmed this in 14 specialist headache centres [7]. Further evaluations are nonetheless needed, and here we conduct a multicentre national study, extending implementation to the quality assessment of six Italian specialist headache centres (level three according to the EHF/LTB standard [8]) and examining acceptability and ease of use of the assessment instrument in another language and country.

\section{Methods}

\section{Participating centres}

Of more than 80 headache specialist centres in Italy, ten participate in a network (the Young Italian Headache Network) of young Italian researchers on headache; seven of these expressed their willingness to participate in the study. However, one centre discontinued its participation for unknown reasons, without contributing any data. The study was completed over 3 months, between September and December 2016, in the remaining six centres (Table 1). The centre of Roma Sapienza, involved also in the earlier study of Schramm et al. [7], collected new data in accordance with our study protocol.

\section{Participants}

In each centre we collected data from service records (via the service manager), from the physicians and other HCPs (nurses, psychologists and/or nursing assistants) involved in outpatient consultations, and from 60 consecutive patients (30 enrolled when making a first-time visit and 30 others undergoing a follow-up visit).

\section{Data collection, and instruments}

We employed the five evaluation instruments used by Schramm et al. [7]. Table 2, set out similarly as in the earlier studies $[6,7]$, provides an overview of these. They included three different questionnaires, one each for the physicians, other HCPs and patients, all completed anonymously. The questionnaire for patients was formulated as an exit questionnaire, which they received from their HCPs at the end of their consultations and completed and returned before leaving. All questionnaires were translated from their English originals into Italian by two authors (LP and SG) according to Lifting The Burden's translation protocol for hybrid documents [9], with TJS and physicians and patients not involved in the study providing review and assistance.

In addition, data were extracted into two datacollection forms from the patients' medical records and from service records. This was done by a local investigator at each centre who, to avoid any bias, did not perform any consultations with patients enrolled in the study. 
Table 1 Characteristics of participating headache centres

\begin{tabular}{|c|c|c|c|c|c|c|c|}
\hline \multirow{2}{*}{\multicolumn{2}{|c|}{ Place }} & \multirow[t]{2}{*}{ Centre name } & \multirow[t]{2}{*}{ Level $^{a}$} & \multirow[t]{2}{*}{ Service description } & \multicolumn{3}{|c|}{ Participants (n) } \\
\hline & & & & & Physicians & $\begin{array}{l}\text { Other } \\
\text { HCPs }\end{array}$ & Patients \\
\hline 1. & Bologna & $\begin{array}{l}\text { Centro per lo Studio e la Cura delle } \\
\text { Cefalee e delle Algie Facciali - Istituto } \\
\text { delle Scienze Neurologiche di Bologna }\end{array}$ & 3 & $\begin{array}{l}\text { Expert advice and care for patients with } \\
\text { headache disorders and facial pain; } \\
\text { headache research and teaching }\end{array}$ & 3 & 6 & 60 \\
\hline 2. & Firenze & $\begin{array}{l}\text { Centro Cefalee e Farmacologia Clinica - } \\
\text { AOUC Università degli studi di Firenze }\end{array}$ & 3 & $\begin{array}{l}\text { University-based service and training centre } \\
\text { run by headache experts; national referral } \\
\text { centre for patients with refractory or rare } \\
\text { headache disorders }\end{array}$ & 4 & 2 & 60 \\
\hline 3. & Modena & $\begin{array}{l}\text { Centro Cefalee ed Abuso di Farmaci - } \\
\text { Policlinico di Modena }\end{array}$ & 3 & $\begin{array}{l}\text { University-based service run by headache- } \\
\text { experienced pharmacologists supported } \\
\text { by } 2 \text { nurses and one consultant psychiatrist }\end{array}$ & 6 & 2 & 60 \\
\hline 4. & Padova & $\begin{array}{l}\text { Dipartimento delle Specialità Mediche - } \\
\text { Neurologia - Centro Cefalee }\end{array}$ & 3 & $\begin{array}{l}\text { Tertiary care centre for diagnosing and } \\
\text { managing headache and other neurological } \\
\text { disorders }\end{array}$ & 2 & 3 & 60 \\
\hline 5. & $\begin{array}{l}\text { Roma Campus } \\
\text { Bio-Medico }\end{array}$ & $\begin{array}{l}\text { Centro per le Cefalee - Campus } \\
\text { Bio-Medico di Roma }\end{array}$ & 3 & $\begin{array}{l}\text { University-based neurological service provided } \\
\text { by physicians experienced in headache } \\
\text { disorders, cerebrovascular diseases and } \\
\text { dementias }\end{array}$ & 5 & 0 & 60 \\
\hline 6. & Roma Sapienza & $\begin{array}{l}\text { Centro di Riferimento Regionale per le } \\
\text { Cefalee - Azienda Ospedaliera } \\
\text { Sant'Andrea di Roma }\end{array}$ & 3 & $\begin{array}{l}\text { University-based centre with daily outpatient, } \\
\text { inpatient and ER service, supported by one } \\
\text { psychologist and two nurses }\end{array}$ & 5 & 3 & 60 \\
\hline
\end{tabular}

according to the EHF/LTB standard [8]

\section{Data management and analysis}

Data were entered into spreadsheets at each centre, and then transferred to the data collection centre (Headache and Drug Abuse Centre, University of Modena and Reggio Emilia).

Descriptive analysis was performed by LP. Demographic and clinical data were provided as numerical values and are summarized as percentages or mean values. No hyphotheses were statistically tested.

\section{Results}

The characteristics of the study centres and participants (physicians, other HCPs and patients) are summarized in Table 1. We took data from a total of 360 patients and from two to six physicians and up to six other HCPs per centre. In one centre (Modena), one physician refused to complete the questionnaire; in all other cases, both HCPs and patients reported that the questionnaires were easy to apply, readily understood and not unduly time consuming. None of the indicators caused or led to difficulties on a practical level; the percentage of missing answers was zero.

Evaluation of each service according to the quality indicators is shown in Table 3, with results summarised below by domain.

\section{Domain A: accurate diagnosis}

Diagnoses were recorded using ICHD terminology in $\geq 97 \%$ of cases. All diagnoses at every centre were documented after the first visit and reviewed during follow-up using diagnostic diaries. Temporal duration of the presenting headache disorder was reported in $\geq 88 \%$ of patients' records (Table 3 ).

\section{Domain B: individualized management}

Waiting-list times for first appointment were not documented, but all clinics reported a formal triage system to expedite appointments in case of perceived urgency.

Mean time allocated to patients' visits ranged, according to patients' reports, between 26 (Padova) and $49 \mathrm{~min}$ (Bologna) for the first visit and between 17 (Firenze) and 29 min (Bologna and Roma Campus Bio-Medico) for follow-up visits. Patients' and HCPs' satisfaction with time per visit was $\geq 83 \%$ in all centres.

Frequency of symptoms and treatment plans were properly reported in most cases (respectively $>82$ and $>87 \%$ ). Psychological therapies were available in four of the six centres: in two of these (Roma Campus BioMedico and Roma Sapienza) their prescription was correctly reported in patients' records, while in the other two (Padova and Firenze) these therapies were not prescribed to any patients enrolled in the study. Disability instruments were available in three centres (Padova, Modena and Firenze), but no HCP utilized them regularly. Follow-up calendars were available and recorded future appointment dates in all centres (Table 3).

\section{Domain C: appropriate referral pathways}

Referral pathways were well established in most centres: exceptions were Roma Campus Bio-Medico for primary care and Padova for urgent consultations (Table 3). 
Table 2 Methods of implementation of service quality indicators

\begin{tabular}{|c|c|}
\hline Indicator & Measure \\
\hline \multicolumn{2}{|c|}{ Domain A. Accurate diagnosis is essential for optimal headache care } \\
\hline $\begin{array}{l}\text { A1. Patients are asked about the temporal profile of } \\
\text { their headaches }\end{array}$ & $\begin{array}{l}\text { a) Duration of presenting complaint is recorded in } \\
\text { patient's record (yes/no) }\end{array}$ \\
\hline \multirow[t]{2}{*}{ A2. Diagnosis is according to current ICHD criteria } & a) Diagnosis is recorded in patient's record (yes/no) \\
\hline & b) Diagnostic record uses ICHD terminology (yes/no) \\
\hline A3. A working diagnosis is made at the first visit & $\begin{array}{l}\text { Working diagnosis at first visit is recorded in patient's } \\
\text { record (yes/no) }\end{array}$ \\
\hline $\begin{array}{l}\text { A4. A definitive diagnosis is made at first or } \\
\text { subsequent visit }\end{array}$ & $\begin{array}{l}\text { Definitive diagnosis is recorded in patient's record or, } \\
\text { if not, an appointment for review has been given } \\
\text { (yes/no) }\end{array}$ \\
\hline A5. Diagnosis is reviewed during later follow-up & $\begin{array}{l}\text { Diagnostic review during follow-up is routinely } \\
\text { undertaken (yes/no) }\end{array}$ \\
\hline A6. Diaries are used to support or confirm diagnosis & $\begin{array}{l}\text { The service has a diagnostic diary available and } \\
\text { physicians are aware of its availability (yes/no) }\end{array}$ \\
\hline
\end{tabular}

Means of enquiry

Patients' records

Patients' records

Patients' records

Patients' records

Physicians' questionnaire

Physicians' questionnaire

Domain B. Individualized management is essential for optimal headache care

B1. Waiting-list times for appointments are related to urgency of need

B2. Sufficient time is allocated to each visit for the purpose of good management

B3. Patients are asked about the temporal profile of their headaches

B4. Treatment plans follow evidence-based guidelines, reflecting diagnosis

B5. Treatment plans include psychological approaches to therapy when appropriate

B6. Treatment plans reflect disability assessment

B7. Patients are followed up to ascertain optimal outcome

a) Waiting-list times are recorded in database (yes/no)

b) A formal triage system exists to expedite appointments in cases of perceived urgency (yes/no)

a) Actual time (minutes) per visit is recorded by patient in exit questionnaire: 1st visits and follow-up visits

b) Patient is satisfied ${ }^{a}$ with actual time (yes/not yes)

c) Health-care providers express overall satisfaction (yes/no)

Frequency (or days/month) of symptoms is recorded in patient's record (yes/no)

Prescribed drugs (names, doses and quantities) are recorded in patient's record

a) Access route to psychological therapies exists (yes/no)

b) Utilisation is recorded in patient's record

a) An instrument for disability assessment is available (yes/no) and is appropriate in the setting (yes/no)

b) Disability is recorded in patient's record (yes/no)

a) Follow-up appointment dates appear in central service records (yes/no)

b) A follow-up diary and/or calendar is available (yes/ no)

Domain C. Appropriate referral pathways are essential for optimal headache care

C1. Referral pathway is available from primary to

A usable pathway exists (yes/no) specialist care

C2. Urgent referral pathway is available when necessary

A usable pathway exists (yes/no)

Domain D. Education of patients about their headaches and their management is essential for optimal headache care

D1. Patients are given the information they need to understand their headache and its management

D2. Patients are given appropriate reassurance

Patient is satisfied ${ }^{a}$ with information given (yes/not yes)

Patient is satisfied ${ }^{a}$ with information given (yes/not yes)

Domain E. Convenience and comfort are part of optimal headache care

E1. The service environment is clean and comfortable

E2. The service is welcoming

a) Patient is satisfied ${ }^{a}$ with cleanliness and comfort (yes/not yes)

b) Health-care providers are satisfied with cleanliness and comfort (yes/no)

Patient is satisfied ${ }^{a}$ with welcome (yes/not yes)
Patients' records

Physicians' questionnaire

Patients' questionnaire

Patients' questionnaire

Physicians' and other HCPs' questionnaires

Patients' records

Patients' records

Physicians' questionnaire

Patients' records

Physicians' questionnaire

Patients' records

Central service records

Physician's questionnaire

Physicians' questionnaire

Physicians' questionnaire

Patients' questionnaire

Patients' questionnaire

Patients' questionnaire

Physicians' and other HCPs' questionnaires

Patients' questionnaire 
Table 2 Methods of implementation of service quality indicators (Continued)

\begin{tabular}{|c|c|c|}
\hline \multirow[t]{3}{*}{ E3. Waiting times in the clinic are acceptable } & $\begin{array}{l}\text { a) Waiting time (minutes) per visit is recorded by } \\
\text { patient in exit questionnaire }\end{array}$ & Patients' questionnaire \\
\hline & b) Patient is satisfied ${ }^{a}$ with waiting time (yes/not yes) & Patients' questionnaire \\
\hline & $\begin{array}{l}\text { c) Health-care providers are satisfied with waiting } \\
\text { times (yes/no) }\end{array}$ & $\begin{array}{l}\text { Physicians' and other HCP } \\
\text { questionnaires }\end{array}$ \\
\hline \multicolumn{3}{|c|}{ Domain F. Achieving patient satisfaction is part of optimal headache care } \\
\hline F1. Patients are satisfied with their management & $\begin{array}{l}\text { Patient is satisfied }{ }^{\mathrm{a}} \text { with overall management (yes/not } \\
\text { yes) }\end{array}$ & Patients' questionnaire \\
\hline \multicolumn{3}{|l|}{ Domain G. Optimal headache care is efficient and equitable } \\
\hline $\begin{array}{l}\text { G1. Procedures are followed to ensure resources are not } \\
\text { wasted }\end{array}$ & A protocol to limit wastage exists (yes/no) & Physicians' questionnaire \\
\hline $\begin{array}{l}\text { G2. Costs of the service are measured as part of a } \\
\text { cost-effectiveness policy }\end{array}$ & A record of input costs exists (yes/no) & Physicians' questionnaire \\
\hline G3. There is equal access to headache services for all & A policy to ensure equal access exists (yes/no) & Physicians' questionnaire \\
\hline
\end{tabular}
who need it

Domain $\mathrm{H}$. Outcome assessment is essential in optimal headache care burden (headache frequency, duration and intensity) _ (yes/no)

b) Outcomes according to this measure are recorded in patient's record (yes/no/not applicable)

H2. Outcome measures are based on self-reported disability burden

a) An outcome measure (HALT or similar) is available (yes/no)

b) Outcomes according to this measure are recorded in patient's record (yes/no/not applicable)

H3. Outcome measures are based on self-reported quality of life

a) An outcome measure (WHOQoL or similar) is available (yes/no)

b) Outcomes according to this measure are recorded in patient's record (yes/no/not applicable)

Domain I. Optimal headache care is safe

11. Patients are not over-treated ${ }^{b}$

Prescribed drugs (names, doses and quantities) are recorded in patient's record (yes/no/not applicable)

12. Systems are in place to be aware of serious adverse events $^{c}$

a) Serious adverse events are recorded

b) A protocol exists for reporting serious adverse events (yes/no)
Physicians' questionnaire

Patients' records

Physicians' questionnaire

Patients' records

Physicians' questionnaire

Patients' records

Patients' records

Patients' records

Physicians' questionnaire

ICHD International Classification of Headache Disorders, HURT Headache Under-Response to Treatment questionnaire [13, 14], HALT Headache-Attributed Lost Time questionnaire [11], WHOQoL World Health Organization Quality of Life questionnaire [12]

aPatients' satisfaction was deduced from Likert scales, extending through the options (according to the question): "too little", "about right", "too much"; or "much too long", "too long", "reasonable"; or "very good", "good", "adequate", "poor", "very poor". These were recoded as "yes" or "not yes" for analysis ("adequate" was coded as "yes")

${ }^{b}$ Over-treatment may mean excessive use of drugs presumed able to induce medication overuse headache, overdosage with potentially harmful drugs such as ergotamine or steroids, use of prophylactic drugs for occasional headache or the wrong diagnosis, use of non-evidence-based treatments that are improbably effective

'Serious adverse events are those that cause death, are life-threatening, terminate or put at risk a pregnancy, or cause hospitalization, prolonged illness, disability and/or malignancy

\section{Domain D: education and reassurance of patients}

Most patients (> 85\%) expressed satisfaction with the information given by physicians, and $>82 \%$ declared they had received appropriate reassurance (Table 3 ).

\section{Domain E: convenience and comfort}

Both patients and HCPs found the service environment clean and comfortable in four centres, with some dissatisfaction expressed in two (Bologna and Firenze). Patients' satisfaction was generally high with the welcome they received from the services (67-98\%) and with waiting times to be seen in the centres (67-82\%). Average waiting times varied little, 8-15 min for first visits and 5-25 min for follow-up visits, and were unsatisfactory only for the HCPs in one centre (Bologna) (Table 3).

Domain F: patient satisfaction

Overall satisfaction with their management was expressed by $78-95 \%$ of patients. (Table 3 ). 
Table 3 Outcomes of implementation of quality indicators by centre (\% of positive answers)

\begin{tabular}{|c|c|c|c|c|c|c|}
\hline & Bologna & Firenze & Modena & Padova & $\begin{array}{l}\text { Roma Campus } \\
\text { Bio-Medico }\end{array}$ & $\begin{array}{l}\text { Roma } \\
\text { La Sapienza }\end{array}$ \\
\hline A1. Duration of complaint recorded & 100 & 88 & 98 & 100 & 90 & 100 \\
\hline A2a. Diagnosis recorded & 100 & 98 & 100 & 100 & 98 & 100 \\
\hline A2b. ICHD terminology used & 100 & 98 & 100 & 100 & 97 & 100 \\
\hline A3. Working diagnosis at first visit recorded & 100 & 98 & 100 & 100 & 98 & 100 \\
\hline A4. Definitive diagnosis or appointment for review & 100 & 98 & 100 & 100 & 100 & 100 \\
\hline A5. Routinely diagnostic review during follow-up (physicians) & 100 & 100 & 100 & 100 & 100 & 100 \\
\hline A6. Diagnostic diaries available (physicians) & 100 & 75 & 100 & 100 & 100 & 100 \\
\hline B1a. Waiting-list times are recorded in database & 0 & 0 & 0 & 0 & 0 & 0 \\
\hline B1b. Formal triage system exists (physicians) & 100 & 75 & 100 & 100 & 100 & 100 \\
\hline B2a. Mean time per visit - 1st visits (minutes) & 49 & 34 & 33 & 26 & 33 & 32 \\
\hline B2a. Mean time per visit - follow up visits (minutes) & 29 & 17 & 28 & 22 & 29 & 20 \\
\hline B2b. Satisfaction with time per visit (patients) & 97 & 90 & 87 & 95 & 97 & 92 \\
\hline B2c. Satisfaction with time per visit (physicians + HCPs) & 100 & 83 & 100 & 100 & 100 & 100 \\
\hline B3. Frequency of symptoms is recorded & 100 & 97 & 82 & 100 & 100 & 100 \\
\hline B4. Prescribed drugs (names, doses and quantities) are recorded & 100 & 95 & 87 & 100 & 100 & 100 \\
\hline B5a. Access route to psychological therapies exists (physicians) & 0 & 100 & 0 & 100 & 100 & 100 \\
\hline B5b. Utilisation of psychological therapies is recorded & $\mathrm{n} / \mathrm{a}$ & $\mathrm{n} / \mathrm{a}$ & $\mathrm{n} / \mathrm{a}$ & $\mathrm{n} / \mathrm{a}$ & 100 & 100 \\
\hline B6a. Instrument for disability assessment is available (physicians) & 0 & 75 & 80 & 100 & 0 & 0 \\
\hline B6b. Instrument for disability is appropriate in the setting (physicians) & $\mathrm{n} / \mathrm{a}$ & 75 & 80 & 100 & $\mathrm{n} / \mathrm{a}$ & $\mathrm{n} / \mathrm{a}$ \\
\hline B6c. Disability is recorded & 0 & 0 & 0 & 0 & 0 & 0 \\
\hline B7a. Follow-up appointment dates appear in central service records & yes & yes & yes & yes & yes & yes \\
\hline B7b. Follow-up diary/calendar is available (physicians) & 100 & 100 & 100 & 100 & 100 & 100 \\
\hline C1. Referral pathway from primary care exists (physicians) & 100 & 100 & 100 & 100 & 0 & 100 \\
\hline C2. Urgent referral pathway exists (physicians) & 100 & 100 & 100 & 0 & 100 & 100 \\
\hline D1. Patient is satisfied with information given & 97 & 90 & 85 & 93 & 95 & 97 \\
\hline D2. Patient is satisfied with reassurance given & 85 & 85 & 82 & 87 & 87 & 95 \\
\hline E1a. Patient is satisfied with cleanliness and comfort & 87 & 52 & 80 & 83 & 95 & 92 \\
\hline E1b. Physicians and HCPs are satisfied with cleanliness and comfort & 67 & 50 & 100 & 100 & 100 & 100 \\
\hline E2. Patient is satisfied with welcome & 83 & 68 & 88 & 90 & 98 & 88 \\
\hline E3a. Mean waiting time - 1st visits (minutes) & 15 & 9 & 11 & 10 & 8 & 11 \\
\hline E3a. Mean waiting time - follow up visits (minutes) & 15 & 9 & 20 & 5 & 5 & 25 \\
\hline E3b. Patient is satisfied with waiting times & 70 & 73 & 70 & 73 & 82 & 68 \\
\hline E3c. Physicians and HCPs are satisfied with waiting times & 11 & 50 & 57 & 80 & 80 & 100 \\
\hline F1. Patient is satisfied with overall management & 83 & 80 & 78 & 90 & 95 & 90 \\
\hline G1. Protocol to limit wastage exists (physicians) & 100 & 25 & 100 & 100 & 100 & 100 \\
\hline G2. Record of input costs exists (physicians) & 100 & 50 & 100 & 0 & 100 & 100 \\
\hline G3. Policy to ensure equal access exists (physicians) & 100 & 100 & 100 & 100 & 100 & 100 \\
\hline H1a. An outcome measure (HURT or similar) exists (physicians) & 0 & 0 & 40 & 100 & 0 & 0 \\
\hline H1b. Outcomes are recorded & 0 & 0 & 0 & 100 & 0 & 0 \\
\hline H2a. An outcome measure (HALT or similar) exists (physicians) & 0 & 75 & 80 & 0 & 0 & 0 \\
\hline H2b. Outcomes are recorded & 0 & 0 & 0 & 0 & 0 & 0 \\
\hline H3a. An outcome measure (WHOQoL or similar) exists (physicians) & 0 & 0 & 0 & 0 & 0 & 0 \\
\hline H3b. Outcomes are recorded & 0 & 0 & 0 & 0 & 0 & 0 \\
\hline
\end{tabular}


Table 3 Outcomes of implementation of quality indicators by centre (\% of positive answers) (Continued)

\begin{tabular}{lllllll}
\hline 11. Prescribed drugs (names, doses and quantities) are recorded & 100 & 95 & 87 & 100 & 100 & 100 \\
I2a. Serious adverse events are recorded & 0 & 0 & 0 & 0 & 0 \\
I2b. Protocol for reporting serious adverse events exists (physicians) & 100 & 75 & 100 & 100 & 100 \\
\hline
\end{tabular}

HCPs Health-care providers, ICHD International Classification of Headache Disorders, HURT Headache Under-Response to Treatment questionnaire [13, 14], HALT Headache-Attributed Lost Time questionnaire [11], WHOQoL World Health Organization Quality of Life questionnaire [12]

\section{Domain G: efficiency and equitability}

All clinics had a protocol to avoid wastage of resources, and input costs were regularly recorded, but not all physicians were aware of these in one centre (Firenze) while the information was available to senior management only in another (Padova). All centres provided equal access for all who might need it.

\section{Domain H: outcome assessment}

Outcome assessments were made routinely only in Padova, and were based on symptom burden. Neither disability nor quality of life were assessed routinely at any centre.

\section{Domain I: safety}

All services appeared safe: prescribed drugs were well documented, protocols for reporting serious adverse events (SAEs) existed in each centre and there were no recorded SAEs.

\section{Discussion}

The study was planned as a multicentre national service quality evaluation. It was conducted in six Italian specialist headache centres, and has shown common practices among them. The centres were all university teaching hospitals, with similar settings and levels of care, and operating within a common culture, with one language and in the same health service. The Italian National Health Service (NHS) has some differences between the administrative regions of Italy, but everywhere provides citizens with free access to a general practitioner, a general paediatrician and admission without charge to hospitals. Specialist consultations, as for headache, are provided with a small charge. Specifically in palliative care, Italian law establishes citizens' right of access to pain care centres for both oncological and non-oncological pain.

However, our objective was not to compare the service quality of these centres but to establish, in Italian headache care, the usability, practicality and acceptability of the quality indicators, and their associated assessment instruments, developed by LTB. The six centres all collected data efficiently, found the questionnaires to be readily understood and easily applied, and, importantly, the whole process was not unduly time consuming. These findings accorded with those of the previous studies with similar purpose [6, 7]. Consequently, we believe the indicators can be used by the Italian headache scientific community to identify majority practices while simultaneously recognizing deficiencies, and therefore to set benchmarks against which quality may be judged in future in this country.

We should nonetheless comment on the specific findings. The results were mostly positive in all 30 quality indicators, but a few deficits were uncovered. In domain A, diagnoses utilized current ICHD criteria, and were routinely reviewed during follow-up; diagnostic diaries were regularly used in each centre. The influence of the continuous efforts of Italian scientific societies to facilitate application of the ICHD criteria is gratifyingly in evidence here. In domain $\mathrm{B}$, triage systems were in place to adjust waiting times to urgency of need, an important consideration. Most patients and physicians were satisfied with the time given in consultations, and with waiting times. Follow-up calendars were available in each centre. On the other hand, no centre recorded in service records the average waiting times for a visit, while HCPs perceived that times were too long for highquality care, a consequence of the high numbers of patients seeing the relatively few doctors working in each centre. Psychological therapies were provided by four centres, with different modalities, a disparity probably due to the difficulties in recruiting psychologists in the NHS (often they are private specialists). Surprisingly, despite that headache disorders are collectively the third highest cause of years lived with disability (YLDs) worldwide [10], no centre recorded patients' disability scores. In domain $C$, referral pathways were in place in five of six centres. Importantly, most patients were generally satisfied and felt welcomed, informed and reassured (domains D, E and F). Furthermore, in domain G, every centre achieved well in the use of protocols to avoid wastage of resources and in promoting equitable access, while in domain I, every service appeared safe.

A critical issue emerged in the lack of formal outcome assessment (domain $\mathrm{H}$ ), indicating the need for greater use of, above all, disability and quality of life instruments (HALT [11] and WHOQoL [12], or similar). In two services (Firenze and Modena), even though the physicians were well aware of the availability of validated and easyto-apply instruments, and of their importance, nobody used them during follow-up because of time constraints. 
All centres complained that their use was not practicable because of lack of time for HCPs to administer them. Nonetheless, without formal and standardized outcomes assessment, it was unclear how the centres established the effectiveness of their interventions against headache. One centre (Padova) routinely used a symptom measure similar to HURT [13, 14], and all others employed some measure of the frequency and severity of headache, such as a headache index and/or numerical rating scales.

\section{Potential for benchmarking}

The consensus group developing these indicators foresaw their employment in benchmarking, but deferred exploration of this potential important application [5]. Our findings in these six centres suggest that the indicators may indeed be deployed in setting benchmarks - at least in Italian specialist headache care. They clearly identified majority practice, with some aspects requiring improvement. Majority practice fails to make routine use of formal outcome measures, despite that this is an obvious part good record-keeping, and would appear to be necessary to judge whether best outcomes are achieved. The evident practical (lack-of-time) difficulty in doing this needs to be addressed. It should be recognized that HURT, for example, is intended for selfcompletion [13, 14], and can be brought by patients to follow-up visits already filled in - and not only used for its purpose of guiding treatment (less necessary in specialist care) but also placed in the patients' records. The solution may lie in developing an electronic version, which has not so far been done. In the context of benchmarking, there are aspects of care not currently included in the quality indicators that many might think should be, and which at least deserve to be discussed for the future. One that is clearly relevant is whether diagnostic examinations and the prescriptions of treatments follow evidence-based guidelines $[15,16]$. The consensus group responsible for developing the indicators acknowledged this, but also recognized that assessment of these was highly problematic [5]. A second aspect, not included in the validated set of quality indicators but perhaps important nonetheless, is the non-medical barriers to care erected, before visits, by various impositions on patients (finding and paying for parking space, for example). We asked patients about their satisfaction with regard to this particular aspect, in a similar manner to the other quality indicators ("yes"/"not yes" responses), obtaining positive replies as follows: Firenze $45 \%$, Roma Sapienza 53\%, Modena 68\%, Padova 80\%, Bologna 82\%, Roma Campus Bio-Medico $87 \%$. Although not a validated quality indicator, this (or an adaption of it) might identify additional difficulties encountered by patients in receiving medical care from the specialist centres that are relevant to service quality.

\section{Conclusions}

This Italy-wide survey, the first such study to examine headache service quality indicators, showed in six specialist centres that the indicators are fit for purpose, identifying many commonalities and detecting deficits as a guide to quality improvement.

While it questioned more than 40 health-care workers and 360 patients, it was deliberately restricted to specialist services operating in similar settings. There was purpose in this, since it was the indicators themselves, and their application, that were being assessed. On the other hand, it was a limitation. Among the next steps is to expand their utilization into more varied primary- and secondarycare settings; most headache patients look for treatment in non-specialist care [16]. Although quality may be an absolute attribute, it is not necessarily attainable to perfection, or there may be practical or cost constraints that plead for compromise. Accordingly, benchmarks may be different in primary care, and this is a pressing area of enquiry. We expect that the quality indicators can be applied in primary care, perhaps with some refinement, but this is yet to be demonstrated.

\begin{abstract}
Acknowledgments
This study was conducted as a project of Young Italian Headache Network. The authors are thankful to Luigi Alberto Pini, for his assistance, support and encouragement.

\section{Funding}

This research did not receive any specific grant from funding agencies in the public, commercial, or not-for-profit sectors.

\section{Authors' contributions}

LP and SG conceived the study, collected data from their centre, performed the statistical analysis and drafting the manuscript. SB, VF, CL, EM, AN, MP, MU SC collected data from the remaining centres and assisted with drafting the manuscript. TJS participated in the study coordination and helped to draft the manuscript. All authors read and approved the final manuscript.
\end{abstract}

\section{Competing interests}

The authors declare that they have no competing interests.

\section{Ethics approval and consent to participate}

Each participating centre obtained approval from its local ethics review committee, before commencing the study. Informed consent was obtained from all participants. Data were collected anonymously, and held in accordance with European data-protection legislation.

\section{Publisher's Note}

Springer Nature remains neutral with regard to jurisdictional claims in published maps and institutional affiliations.

\section{Author details}

${ }^{1}$ Medical Toxicology - Headache Centre, Policlinic Hospital, University of Modena and Reggio Emilia, Modena, Italy. ${ }^{2}$ Department of Health Sciences, Section of Clinical Pharmacology and Oncology, University of Florence, Florence, Italy. ${ }^{3}$ IRCCS Institute of Neurological Sciences of Bologna, Department of Biomedical and Neuromotor Sciences DIBINEM, University of Bologna, Bologna, Italy. ${ }^{4}$ Department of Neurosciences, Headache Centre, University of Padua, Padua, Italy. ${ }^{5}$ Department of Clinical and Molecular Medicine, Sapienza University of Rome, Sant'Andrea Hospital, Rome, Italy. ${ }^{6}$ Headache Centre, Neurology Unit, University Campus Bio-Medico, Rome, Italy. ${ }^{7}$ Department of Neuromedicine and Movement Science, NTNU Norwegian University of Science and Technology, Trondheim, Norway. 
${ }^{8}$ Division of Brain Sciences, Imperial College London, London, UK. ${ }^{9}$ RCCS Institute of Neurological Sciences of Bologna, Bologna, Italy.

Received: 12 February 2017 Accepted: 27 April 2017

Published online: 05 May 2017

\section{References}

1. Headache Classification Committee of the International Headache Society (1988) Classification and diagnostic criteria for headache disorders, cranial neuralgias and facial pain. Cephalalgia 8(Suppl 7):1-96

2. Peters M, Perera S, Loder E, Jenkinson C, Gil-Gouveia R, Jensen R, Katsarava Z, Steiner TJ (2012) Quality in the provision of headache care. 1: systematic review of the literature and commentary. J Headache Pain 13:437-447

3. Steiner TJ (2004) Lifting the burden: the global campaign against headache. Lancet Neurol 3(4):204-205

4. Steiner TJ, Birbeck GL, Jensen R, Katsarava Z, Martelletti P, Stovner $\sqcup$ (2010) Lifting The Burden: the first 7 years. J Headache Pain 11(6):451-455

5. Peters M, Jenkinson C, Perera S, Loder E, Jensen R, Katsarava Z, Gil-Gouveia R, Broner S, Steiner TJ (2012) Quality in the provision of headache care. 2: defining quality and its indicators. J Headache Pain 13:449-457

6. Katsarava Z, Gil-Gouveia R, Jensen R, Gaul C, Schramm S, Schoppe A, Steiner TJ (2015) Evaluation of headache service quality indicators: pilot implementation in two specialist-care centres. J Headache Pain 16:53

7. Schramm S, Uluduz D, Gouveia RG et al (2016) Headache service quality: evaluation of quality indicators in 14 specialist-care centers. J Headache Pain 17:111

8. Steiner TJ, Antonaci F, Jensen R, Lainez JMA, Lantèri-Minet M, Valade $D$ (2011) Recomendations for headache service organisation and delivery in Europe. J Headache Pain 12:419-426

9. Peters M, Bertolote JM, Houchin C, Kandoura T, Steiner TJ (2007) Translation protocol for hybrid documents. J Headache Pain 8(suppl 1):S45-S47

10. Steiner TJ, Birbeck GL, Jensen RH, Katsarava Z, Stovner LJ, Martelletti P (2015) Headache disorders are third cause of disability worldwide. J Headache Pain 16:58

11. Steiner TJ (2007) The HALT and HART indices. J Headache Pain 8(suppl 1): S22-S25

12. Schmidt S, Mühlan H, Power M (2005) The EUROHIS-OOL 8-item index: psychometric results of a cross-cultural field study. Eur J Pub Health 16:420-428

13. Buse DC, Sollars CM, Steiner TJ, Jensen RH, Al Jumah MA, Lipton RB (2012) Why HURT? A review of clinical instruments for headache management. Curr Pain Headache Rep 16:237-254

14. Westergaard MLS, Steiner TJ, MacGregor EA, Antonaci F, Tassorelli C, Buse DC, Lipton RB, Jensen RH (2013) The Headache Under-Response to Treatment (HURT) questionnaire: assessment of utility in headache specialist care. Cephalalgia 33:245-255

15. Mitsikostas DD, Ashina M, Craven A, Diener HC, Goadsby PJ, Ferrari MD, Lampl C, Paemeleire K, Pascual J, Siva A, Olesen J, Osipova V, Martelletti P, EHF committee (2015) European headache Federation consensus on technical investigation for primary headache disorders. J Headache Pain 17:5

16. Steiner TJ, Paemeleire K, Jensen R, Valade D, Savi L, Lainez MJ, Diener HC, Martelletti P, Couturier EG (2007) European principles of management of common headache disorders in primary care. J Headache Pain 8(suppl 1):S3-S21

\section{Submit your manuscript to a SpringerOpen ${ }^{\circ}$ journal and benefit from:}

- Convenient online submission

- Rigorous peer review

Immediate publication on acceptance

- Open access: articles freely available online

- High visibility within the field

- Retaining the copyright to your article 